International Journal of Bifurcation and Chaos

(c) World Scientific Publishing Company

\title{
Metastable Systems as Random Maps
}

\author{
Paweł Góra \\ Department of Mathematics and Statistics, Concordia University, \\ 1455 de Maisonneuve Blvd. West, Montreal, Quebec H3G 1M8, Canada \\ pgora@mathstat.concordia.ca \\ Abraham Boyarsky \\ Department of Mathematics and Statistics, Concordia University, \\ 1455 de Maisonneuve Blvd. West, Montreal, Quebec H3G 1M8, Canada \\ boyar@alcor.concordia.ca \\ Peyman Eslami \\ Department of Mathematics and Statistics, Concordia University, \\ 1455 de Maisonneuve Blvd. West, Montreal, Quebec H3G 1M8, Canada \\ peyman_eslami@yahoo.com
}

Received (to be inserted by publisher)

\begin{abstract}
Metastable dynamical systems were recently studied [Gonzáles-Tokman et al., 2011] in the framework of one-dimensional piecewise expanding maps on two disjoint invariant sets, each possessing its own ergodic absolutely continuous invariant measure (acim). Under small deterministic perturbations, holes between the two disjoint systems are created, and the two ergodic systems merge into one. The long term dynamics of the newly formed metastable system is defined by the unique acim on the combined ergodic sets. The main result of [Gonzáles-Tokman et al., 2011] proves that this combined acim can be approximated by a convex combination of the disjoint acims with weights depending on the ratio of the respective measures of the holes. In this note we present an entirely different approach to metastable systems. We consider two piecewise expanding maps: one is the original map, $\tau_{1}$, defined on two disjoint invariant sets of $\mathbb{R}^{N}$ and the other is a deterministically perturbed version of $\tau_{1}, \tau_{2}$, which allows passage between the two disjoint invariant sets of $\tau_{1}$. We model this system by a position dependent random map based on $\tau_{1}$ and $\tau_{2}$, to which we associate position dependent probabilities that reflect the switching between the maps. A typical orbit spends a long time in one of the ergodic sets but eventually switches to the other. Such behavior can be attributed to physical holes as between adjoining billiard tables or more abstract situations where balls can "leap" from one table to the other. Using results for random maps a result similar to the one dimensional main result of [Gonzáles-Tokman et al., 2011] is proved in $N$ dimensions. We also consider holes in more than two invariant sets. A number of examples are presented.
\end{abstract}

Keywords: metastable systems, absolutely continuous invariant measures, random maps

\section{Introduction}

One-dimensional metastable systems were recently studied [Gonzáles-Tokman et al., 2011] in the framework of piecewise expanding maps on two disjoint ergodic sets. Under small deterministic perturbations, the 
asymptotic dynamics of the merged metastable system is captured by the absolutely continuous invariant measure (acim) on the combined ergodic sets. The main result of [Gonzáles-Tokman et al., 2011] shows that this combined acim can be approximated by a convex combination of the two disjoint acims with weights depending on the respective measures of the holes. The method of [Gonzáles-Tokman et al., 2011] invokes the usual BV techniques that apply naturally in a setting where the slopes of the original map are $>2$. For maps with slopes only $>1$ in magnitude, the BV technique encounters difficulties as the partitions needed for the approximating family of maps have elements that go to zero in measure and hence render the standard BV inequalities ineffective in establishing precompactness of the family of probability density functions associated with the family of approximating maps. To handle this problem, the authors of [Gonzáles-Tokman et al., 2011] introduce some additional conditions on the maps they consider.

In this note we take a different approach to modeling metastable behavior. We consider two piecewise expanding maps: one is the original map, $\tau_{1}$, defined on two disjoint invariant sets of $\mathbb{R}^{N}$ and the other, $\tau_{2}$, is a deterministically perturbed version of $\tau_{1}, \tau_{2}$, which allows passage between the two disjoint invariant sets of $\tau_{1}$ via holes. We model such a system by means of a random map based on $\tau_{1}$ and $\tau_{2}$, to which we associate position dependent probabilities that reflect the switching between the maps. A typical orbit spends a random amount of time governed by the dynamics of either $\tau_{1}$ or $\tau_{2}$, then switches to the other map. Suppose $p_{1}$, the probability of using map $\tau_{1}$, is close to 1 , then, with very high probability, the orbit spends a lot of time under the influence of $\tau_{1}$, that is, it stays in either one or the other of the two disjoint sets invariant under $\tau_{1}$. Since $p_{1}<1$, there is a small but positive probability of switching from $\tau_{1}$ to $\tau_{2}$. When this happens, the dynamics comes under the control of $\tau_{2}$, which allows movement between the disjoint invariant sets. Unlike the model in [Gonzáles-Tokman et al., 2011] where the hole sizes shrink to 0 , the hole sizes in our random map model stay fixed. (Their measures in a skew product interpretation of random maps converge to 0 , so one could argue that both models are in a way similar.) What changes are the probabilities of switching from one map to the other. As $p_{1}$ approaches 1, the orbits are almost completely defined by $\tau_{1}$ and therefore remain in one or the other of the two disjoint invariant sets for a very long time. This behavior is the hallmark of metastable dynamics. Our main result establishes a result similar in spirit to that in [Gonzáles-Tokman et al., 2011]: we prove that, as the probability of using $\tau_{1}$ converges to 1 , the dynamics are captured by an acim that is a convex combination of the acims on the two disjoint invariant sets and we can calculate the weights of the respective acims from a formula analogous to the one derived in [Gonzáles-Tokman et al., 2011].

In the billiards problem metastable behavior is attributed to small physical holes in the boundary between the tables. From the perspective of random maps, the holes can be large; it is the probabilities of switching that controls the metastable behavior. This allows for the consideration of situations where there are no actual physical holes, but where balls can "leap" from one table to the other.

In Sections 2 and 3 we recall the definition of a position dependent random map and collect some existence and continuity results in 1 and $N$ dimensions. In Section 4 we present the main result for holes between two invariant sets: there exists a unique acim which is a convex combination of the acims on the two ergodic sets and the weights in the combination can be calculated from a formula similar to the one in [Gonzáles-Tokman et al., 2011]. In Section 5 we present the main result for a case with more than two invariant subsystems. Deterministic model of such situation is discussed in [Dolgopyat and Wright, 2011]. Section 6 contains examples.

\section{Position Dependent Random Maps and Their Properties}

Let $(I, \mathfrak{B}, \lambda)$ be a measure space, where $\lambda$ is an underlying measure. Let $\tau_{k}: I \rightarrow I, k=1, \ldots, K$ be piecewise one-to-one, differentiable, non-singular transformations on a common partition $\mathcal{P}$ of $I: \mathcal{P}=\left\{I_{1}, \ldots, I_{q}\right\}$ and $\tau_{k, i}=\left.\tau_{k}\right|_{I_{i}}, i=1, \ldots, q, k=1, \ldots, K(\mathcal{P}$ can be found by considering finer partitions). We define the transition function for the random map $T=\left\{\tau_{1}, \ldots \tau_{K} ; p_{1}(x), \ldots p_{K}(x)\right\}$ as follows:

$$
\mathbb{P}(x, A)=\sum_{k=1}^{K} p_{k}(x) \chi_{A}\left(\tau_{k}(x)\right)
$$


where $A$ is any measurable set and $\left\{p_{k}(x)\right\}_{k=1}^{K}$ is a set of position dependent measurable probabilities, i.e., $\sum_{k=1}^{K} p_{k}(x)=1, p_{k}(x) \geq 0$, for any $x \in I$ and $\chi_{A}$ denotes the characteristic function of the set $A$. We define $T(x)=\tau_{k}(x)$ with probability $p_{k}(x)$ and $T^{N}(x)=\tau_{k_{N}} \circ \tau_{k_{N-1}} \circ \ldots \circ \tau_{k_{1}}(x)$ with probability $p_{k_{N}}\left(\tau_{k_{N-1}} \circ \ldots \circ \tau_{k_{1}}(x)\right) \cdot p_{k_{N-1}}\left(\tau_{k_{N-2}} \circ \ldots \circ \tau_{k_{1}}(x)\right) \cdots p_{k_{1}}(x)$. The transition function $\mathbb{P}$ induces an operator $\mathbb{P}_{*}$ on measures on $(I, \mathfrak{B})$ defined by

$$
\begin{aligned}
\mathbb{P}_{*} \mu(A) & =\int_{I} \mathbb{P}(x, A) d \mu(x)=\sum_{k=1}^{K} \int_{I} p_{k}(x) \chi_{A}\left(\tau_{k}(x)\right) d \mu(x) \\
& =\sum_{k=1}^{K} \int_{\tau_{k}^{-1}(A)} p_{k}(x) d \mu(x)=\sum_{k=1}^{K} \sum_{i=1}^{q} \int_{\tau_{k, i}^{-1}(A)} p_{k}(x) d \mu(x)
\end{aligned}
$$

We say that the measure $\mu$ is $T$-invariant iff $\mathbb{P}_{*} \mu=\mu$, i.e.,

$$
\mu(A)=\sum_{k=1}^{K} \int_{\tau_{k}^{-1}(A)} p_{k}(x) d \mu(x), \quad A \in \mathfrak{B} .
$$

If $\mu$ has density $f$ with respect to $\lambda$, the $\mathbb{P}_{*} \mu$ has also a density which we denote by $P_{T} f$. By change of variables, we obtain

$$
\begin{aligned}
\int_{A} P_{T} f(x) d \lambda(x) & =\sum_{k=1}^{K} \sum_{i=1}^{q} \int_{\tau_{k, i}^{-1}(A)} p_{k}(x) f(x) d \lambda(x) \\
& =\sum_{k=1}^{K} \sum_{i=1}^{q} \int_{A} p_{k}\left(\tau_{k, i}^{-1} x\right) f\left(\tau_{k, i}^{-1} x\right) \frac{1}{J_{k, i}\left(\tau_{k, i}^{-1}\right)} d \lambda(x),
\end{aligned}
$$

where $J_{k, i}$ is the Jacobian of $\tau_{k, i}$ with respect to $\lambda, J(\tau)=\frac{d \tau_{*}(\lambda)}{d \lambda}$. Since this holds for any measurable set $A$ we obtain an a.e. equality:

$$
\left(P_{T} f\right)(x)=\sum_{k=1}^{K} \sum_{i=1}^{q} p_{k}\left(\tau_{k, i}^{-1} x\right) f\left(\tau_{k, i}^{-1} x\right) \frac{1}{J_{k, i}\left(\tau_{k, i}^{-1}\right)} \chi_{\tau_{k}\left(I_{i}\right)}(x)
$$

or

$$
\left(P_{T} f\right)(x)=\sum_{k=1}^{K} P_{\tau_{k}}\left(p_{k} f\right)(x)
$$

where $P_{\tau_{k}}$ is the Perron-Frobenius operator corresponding to the transformation $\tau_{k}$ (see [Boyarsky and Góra, 1997] for details). We call $P_{T}$ the Perron-Frobenius operator of the random map $T$.

\section{Continuity theorems}

\subsection{Existence and continuity theorem in one dimension}

Let $(I, \mathfrak{B}, \lambda)$ be a measure space, where $\lambda$ is normalized Lebesgue measure on $I=[a, b]$. Let $\tau_{k}: I \rightarrow I$, $k=1, \ldots, K$ be piecewise one-to-one and $C^{2}$, non-singular transformations on a partition $\mathcal{P}$ of $I: \mathcal{P}=$ $\left\{I_{1}, \ldots, I_{q}\right\}$ and $\tau_{k, i}=\left.\tau_{k}\right|_{I_{i}}, i=1, \ldots, q, k=1, \ldots, K$. Let $\left\{p_{k}(x)\right\}_{k=1}^{K}$ be a set of position dependent measurable probabilities, i.e., $\sum_{k=1}^{K} p_{k}(x)=1, p_{k}(x) \geq 0$, for any $x \in I$. Assume in addition that $p_{k}$ is piecewise differentiable on $\mathcal{P}$.

Denote by $V(\cdot)$ the standard one-dimensional variation of a function, and by $B V(I)$ the space of functions of bounded variations on $I$ equipped with the norm $\|\cdot\|_{B V}=V(\cdot)+\|\cdot\|_{1}$.

Let $g_{k}(x)=\frac{p_{k}(x)}{\left|\tau_{k}^{\prime}(x)\right|}, k=1, \ldots, K$. We assume the following conditions:

Condition (A): $\sum_{k=1}^{K} g_{k}(x)<\alpha<1, x \in I$, and

Condition (B): $g_{k} \in B V(I), k=1, \ldots, K$. 
Let $T=\left\{\tau_{1}, \ldots, \tau_{K} ; p_{1}, \ldots, p_{K}\right\}$ be a random map with position dependent probabilities satisfying conditions $(A)$ and $(B)$. We define $\mathcal{P}^{N}$ as a maximal common monotonicity partition for all maps defining $T^{N}$. For $w=\left(k_{1}, \ldots, k_{N-1}, k_{N}\right) \in\{1, \ldots, K\}^{N}$ we define

$$
g_{w}=\frac{p_{k_{N}}\left(\tau_{k_{N-1}} \circ \ldots \circ \tau_{k_{1}}(x)\right) \cdot p_{k_{N-1}}\left(\tau_{k_{N-2}} \circ \ldots \circ \tau_{k_{1}}(x)\right) \cdots p_{k_{1}}(x)}{\left|\left(\tau_{k_{N}} \circ \tau_{k_{N-1}} \circ \ldots \circ \tau_{k_{1}}\right)^{\prime}(x)\right|} .
$$

The following results are proved in [Bahsoun and Góra, 2005]:

Lemma 1. Let $T$ satisfy conditions $(A)$ and $(B)$. Then for any $f \in B V(I)$ and $M \in \mathbb{N}$,

$$
\left\|P_{T}^{M} f\right\|_{B V} \leq A_{M}\|f\|_{B V}+B_{M}\|f\|_{1}
$$

where $A_{M}=3 \alpha^{M}+W_{M}, B_{M}=\beta_{M}\left(2 \alpha^{M}+W_{M}\right), \quad \beta_{M}=\max _{J \in \mathcal{P}^{M}}(\lambda(J))^{-1}, W_{M} \equiv$ $\max _{J \in \mathcal{P}^{M}} \sum_{w \in\{1, \ldots, K\}^{M}} V_{J} g_{w}$.

Theorem 1. Let $T$ be a random map which satisfies conditions (A) and (B). Then $T$ preserves a measure which is absolutely continuous with respect to Lebesgue measure. The operator $P_{T}$ is quasi-compact on $B V(I)$, see [Boyarsky and Góra, 1997].

We now present the continuity theorem in one dimension. A similar theorem was proved in proposition 2 of [Góra and Boyarsky, 2003] under stronger conditions. Our aim is to show that it holds under the weaker conditions $(A)$ and $(B)$.

Theorem 2 [Continuity Theorem 1-dim]. Let $T=\left\{\tau_{1}, \ldots, \tau_{K} ; p_{1}, \ldots, p_{K}\right\}$ be a random map with position dependent probabilities satisfying conditions $(A)$ and $(B)$. Let $\left\{p_{1}^{(n)}, \ldots, p_{K}^{(n)}\right\}_{n=1}^{\infty}$ be a sequence of sets of probabilities such that $p_{k}^{(n)} \rightarrow p_{k}$ as $n \rightarrow+\infty, k=1, \ldots, K$, in the piecewise $C^{1}$ topology on the fixed partition $\mathcal{P}$. Let $T^{(n)}=\left\{\tau_{1}, \ldots, \tau_{K} ; p_{1}^{(n)}, \ldots, p_{K}^{(n)}\right\}, n=1,2, \ldots$ be a sequence of random maps. For $n$ large, $T^{(n)}$ has an invariant density $f^{(n)}$ and the sequence $\left\{f^{(n)}\right\}_{n=1}^{\infty}$ is precompact in $L^{1}$. Moreover, any limit point $f^{*}$ of this sequence is a fixed point of $P_{T}$.

Proof. We will prove the theorem in three steps. In the first step we show that an inequality similar to inequality (7) of lemma 1 holds uniformly for all $T^{(n)}$ with $n$ large enough. In order to achieve this, we need to show that for large enough $n$ conditions $(A)$ and $(B)$ are satisfied uniformly.

Suppose $\alpha<\gamma<1$, where $\sum_{k=1}^{K} g_{k}(x)<\alpha<1$. First, choose $\epsilon$ such that $\sum_{k=1}^{K} \frac{\epsilon}{\left|\tau_{k}^{\prime}(x)\right|}<\gamma-\alpha$. Then choose $N_{1}$ such that for $n>N_{1}$ and $1 \leq k \leq K, p_{k}-\epsilon \leq p_{k}^{(n)} \leq p_{k}+\epsilon$. Then

$$
\sum_{k=1}^{K} \frac{p_{k}^{(n)}(x)}{\left|\tau_{k}^{\prime}(x)\right|} \leq \sum_{k=1}^{K} \frac{p_{k}+\epsilon}{\left|\tau_{k}^{\prime}(x)\right|}=\sum_{k=1}^{K} \frac{p_{k}(x)}{\left|\tau_{k}^{\prime}(x)\right|}+\sum_{k=1}^{K} \frac{\epsilon}{\left|\tau_{k}^{\prime}(x)\right|} \leq \alpha+(\gamma-\alpha)=\gamma<1 .
$$

Therefore, condition (A) holds uniformly for all $n>N_{1}$, with $\alpha$ replaced by $\gamma$. Regarding condition (B), note that

$$
\left|V_{J} g^{(n)}-V_{J} g\right| \leq \int_{J}\left|\left(g^{(n)}\right)^{\prime}-g^{\prime}\right| d \lambda \rightarrow 0 \text { as } n \rightarrow \infty .
$$

It follows that there exists a constant $C_{1}$ and an integer $N_{2}$ such that for all $n>N_{2}, V_{J} g^{(n)}<C_{1}$ for any interval $J \subset I$.

Now consider $W_{1}^{(n)}=\max _{J \in \mathcal{P}} \sum_{k=1}^{K} V_{J} g_{k}^{(n)}$. From the above statement it follows that $W_{1}^{(n)}$ is also uniformly bounded for $n$ sufficiently large. That is, there exists $C_{2}$ and integer $N_{3}$ such that for all $n>N_{3}$, $W_{1}^{(n)}<C_{2}$. Let $N_{4}=\max \left\{N_{1}, N_{2}, N_{3}\right\}$ and $C=\max \left\{C_{1}, C_{2}\right\}$. It is shown in [Bahsoun and Góra, 2005] that $W_{M}^{(n)} \leq M \alpha^{M-1} W_{1}^{(n)}$, hence for $n>N_{4}, W_{M}^{(n)}<M \gamma^{M-1} C$. Therefore, for $n>N_{4}$, inequality (7) holds uniformly with $\alpha$ replaced by $\gamma$.

In the next step we show that the sequence of invariant densities $\left\{f^{(n)}\right\}$ is uniformly bounded in $B V(I)$. Without loss of generality consider $\left\{f^{(n)}\right\}_{n=N_{4}+1}^{\infty}$ instead of $\left\{f^{(n)}\right\}$. Moreover since inequality (7) is now 
satisfied uniformly for all $n$, we drop the superscript of $(n)$ and write $A_{M}, B_{M}$ for $A_{M}^{(n)}, B_{M}^{(n)}$ respectively. Also assume $M$ is large enough so that $A_{M}=3 \gamma^{M}+W_{M}<1$.

To summarize, we have shown that there exists $M$ such that for any $f \in B V(I)$ and $n \in \mathbb{N}$ :

$$
\left\|P_{T^{(n)}}^{M} f\right\|_{B V} \leq A_{M}\|f\|_{B V}+B_{M}\|f\|_{1},
$$

where $A_{M}=3 \gamma^{M}+W_{M}<1, B_{M}=\beta_{M}\left(2 \gamma^{M}+W_{M}\right), \beta_{M}=\max _{J \in \mathcal{P}^{M}}(\lambda(J))^{-1}, W_{M} \equiv$ $\max _{J \in \mathcal{P}^{M}} \sum_{w \in\{1, \ldots, K\}^{M}} V_{J} g_{w}$.

Using inequality (8) repeatedly, one can show that each $f^{(n)}$ is a limit point of the sequence of averages $\left\{\frac{1}{m} \sum_{j=0}^{m-1} P_{T^{(n)}}^{M j} 1\right\}$ and

$$
\left\|f^{(n)}\right\|_{B V} \leq 1+\frac{B_{M}}{1-A_{M}} .
$$

Therefore $\left\{f^{(n)}\right\}$ is a bounded set in $B V(I)$ and hence it has a limit point $f^{*}$ in $L^{1}$.

In the final step show that $f^{*}$ is invariant under $P_{T}$ :

$$
\begin{aligned}
\left\|P_{T} f^{*}-f^{*}\right\|_{1} & \leq\left\|P_{T} f^{*}-P_{T^{(n)}} f^{*}\right\|_{1}+\left\|P_{T^{(n)}} f^{*}-P_{T^{(n)}} f^{(n)}\right\|_{1} \\
& +\left\|P_{T^{(n)}} f^{(n)}-f^{(n)}\right\|_{1}+\left\|f^{(n)}-f^{*}\right\|_{1} \\
& =\left\|\sum_{k=1}^{K} P_{\tau_{k}}\left(p_{k} f^{*}\right)-\sum_{k=1}^{K} P_{\tau_{k}}\left(p_{k}^{(n)} f^{*}\right)\right\|_{1} \\
& +\left\|\sum_{k=1}^{K} P_{\tau_{k}}\left(p_{k}^{(n)} f^{*}\right)-\sum_{k=1}^{K} P_{\tau_{k}}\left(p_{k}^{(n)} f^{(n)}\right)\right\|_{1} \\
& +\left\|P_{T^{(n)}} f^{(n)}-f^{(n)}\right\|_{1}+\left\|f^{(n)}-f^{*}\right\|_{1} \\
& \leq \sum_{k=1}^{K}\left\|f^{*}\left(p_{k}-p_{k}^{(n)}\right)\right\|_{1}+\sum_{k=1}^{K}\left\|\left(f^{*}-f^{(n)}\right) p_{k}^{(n)}\right\|_{1} \\
& +\left\|P_{T^{(n)}} f^{(n)}-f^{(n)}\right\|_{1}+\left\|f^{(n)}-f^{*}\right\|_{1}
\end{aligned}
$$

The third summand is 0 by definition of $f^{(n)}$. The other three converge to 0 since $f^{(n)} \rightarrow f^{*}$ and $p_{k}^{(n)} \rightarrow p_{k}$ as $n \rightarrow \infty$ in $L^{1}$ and $L^{\infty}$, respectively.

\subsection{Existence and continuity theorem in higher dimensions}

We now prove the continuity theorem in $\mathbb{R}^{N}$. Let $S$ be a bounded region in $\mathbb{R}^{N}$ and $\lambda_{N}$ be Lebesgue measure on $S$. Let $\tau_{k}: S \rightarrow S, k=1, \ldots, K$ be piecewise one-to-one and $C^{2}$, non-singular transformations on a partition $\mathcal{P}$ of $S: \mathcal{P}=\left\{S_{1}, \ldots, S_{q}\right\}$ and $\tau_{k, i}=\left.\tau_{k}\right|_{S_{i}}, i=1, \ldots, q, k=1, \ldots, K$. Let each $S_{i}$ be a bounded closed domain having a piecewise $C^{2}$ boundary of finite $(N-1)$-dimensional measure. We assume that the faces of $\partial S_{i}$ meet at angles bounded uniformly away from 0 . We will also assume that the probabilities $p_{k}(x)$ are piecewise $C^{1}$ functions on the partition $\mathcal{P}$. Let $D \tau_{k, i}^{-1}(x)$ be the derivative matrix of $\tau_{k, i}^{-1}$ at $x$. We assume:

\section{Condition (C):}

$$
\max _{1 \leq i \leq q} \sum_{k=1}^{K} p_{k}(x)\left\|D \tau_{k, i}^{-1}\left(\tau_{k, i}(x)\right)\right\|<\sigma<1 .
$$

The main tool of this section is the multidimensional notion of variation defined using derivatives in the distributional sense (see [Giusti, 1984]):

$$
V(f)=\int_{\mathbb{R}^{N}}\|D f\|=\sup \left\{\int_{\mathbb{R}^{N}} f \operatorname{div}(g) d \lambda_{N}: g=\left(g_{1}, \ldots, g_{N}\right) \in C_{0}^{1}\left(\mathbb{R}^{N}, \mathbb{R}^{N}\right)\right\},
$$


where $f \in L_{1}\left(\mathbb{R}^{N}\right)$ has bounded support, $D f$ denotes the gradient of $f$ in the distributional sense, and $C_{0}^{1}\left(\mathbb{R}^{N}, \mathbb{R}^{N}\right)$ is the space of continuously differentiable functions from $\mathbb{R}^{N}$ into $\mathbb{R}^{N}$ having a compact support. We will use the following property of variation which is derived from [Giusti, 1984], Remark 2.14: If $f=0$ outside a closed domain $A$ whose boundary is Lipschitz continuous, $f_{\mid A}$ is continuous, $f_{\mid \operatorname{int}(A)}$ is $C^{1}$, then

$$
V(f)=\int_{\operatorname{int}(A)}\|D f\| d \lambda_{N}+\int_{\partial A}|f| d \lambda_{N-1}
$$

where $\lambda_{N-1}$ is the $(N-1)$-dimensional measure on the boundary of $A$. In this section we shall consider the Banach space (see [Giusti, 1984], Remark 1.12),

$$
B V(S)=\left\{f \in L_{1}(S): V(f)<+\infty\right\},
$$

with the norm $\|f\|_{B V}=V(f)+\|f\|_{1}$.

Theorems 3 and 4 below were established in [Góra and Boyarsky, 1989]. We refer the reader to [Góra and Boyarsky, 1989] for proofs as well as the precise definitions of the functions $a(\cdot)$ and $\delta(\cdot)$. We just remark here that for a random map $T=\left\{\tau_{1}, \ldots, \tau_{K} ; p_{1}, \ldots, p_{K}\right\}$ the functions $a$ and $\delta$ are independent of the probabilities $\left\{p_{1}, \ldots, p_{K}\right\}$.

Theorem 3. If $T$ is a random map which satisfies condition $(C)$, then

$$
V\left(P_{T} f\right) \leq \sigma(1+1 / a) V(f)+\left(M+\frac{\sigma}{a \delta}\right)\|f\|_{1},
$$

where $a=\min \left\{a\left(S_{i}\right): i=1, \ldots, q\right\}>0, \delta=\min \left\{\delta\left(S_{i}\right),: i=1, \ldots, q\right\}>0, M_{k, i}=\sup _{x \in S_{i}}\left(D p_{k}(x)-\right.$ $\left.\frac{D J_{k, i}}{J_{k, i}} p_{k}(x)\right)$ and $M=\sum_{k=1}^{K} \max _{1 \leq i \leq q} M_{k, i}$.

Theorem 4. Let $T$ be a random map which satisfies condition $(C)$. If $\sigma(1+1 / a)<1$, then $T$ preserves a measure which is absolutely continuous with respect to Lebesgue measure. The operator $P_{T}$ is quasi-compact on $B V(S)$, see [Boyarsky and Góra, 1997].

Now we present the multi-dimensional version of theorem 2. The proof of this theorem is similar to the proof of the one-dimensional continuity theorem hence we will only sketch the proof here.

Theorem 5 [Continuity Theorem in $\left.\mathbb{R}^{N}\right]$. Let $T=\left\{\tau_{1}, \ldots, \tau_{K} ; p_{1}, \ldots, p_{K}\right\}$ be a random map with position dependent probabilities, satisfying condition $(C)$. Also assume that $\sigma(1+1 / a)<1$, where a is as in [Bahsoun and Góra, 2005]. Let $\left\{p_{1}^{(n)}, \ldots, p_{K}^{(n)}\right\}_{n=1}^{\infty}$ be a sequence of sets of probabilities such that $p_{k}^{(n)} \rightarrow p_{k}$ as $n \rightarrow+\infty, k=1, \ldots, K$, in the piecewise $C^{1}$ topology on the fixed partition $\mathcal{P}$. Let $T^{(n)}=\left\{\tau_{1}, \ldots, \tau_{K} ; p_{1}^{(n)}, \ldots, p_{K}^{(n)}\right\}, n=1,2, \ldots$ be a sequence of random maps. For $m$ large, $T^{(n)}$ has an invariant density $f^{(n)}$ and the sequence $\left\{f^{(n)}\right\}_{n=1}^{\infty}$ is precompact in $L^{1}$. Moreover, any limit point $f^{*}$ of this sequence is a fixed point of $P_{T}$.

Proof. The main part of the proof is to establish an inequality similar to inequality (9) uniformly for all $n$ larger than some integer $N_{1}$. As a result of applying theorem 3 to $T^{(n)}$ we obtain:

$$
V\left(P_{T^{(n)}} f\right) \leq \underbrace{\sigma^{(n)}\left(1+1 / a^{(n)}\right)}_{A^{(n)}} V(f)+\underbrace{\left(M^{(n)}+\frac{\sigma^{(n)}}{a^{(n)} \delta}\right.}_{B^{(n)}})\|f\|_{1},
$$

where $a^{(n)}=\min \left\{a^{(n)}\left(S_{i}\right): i=1, \ldots, q\right\}>0, \delta^{(n)}=\min \left\{\delta\left(S_{i}\right),: i=1, \ldots, q\right\}>0, M_{k, i}^{(n)}=$ $\sup _{x \in S_{i}}\left(D p_{k}^{(n)}(x)-\frac{D J_{k, i}}{J_{k, i}} p_{k}^{(n)}(x)\right)$ and $M^{(n)}=\sum_{k=1}^{K} \max _{1 \leq i \leq q} M_{k, i}^{(n)}$.

Note that $a^{(n)}$ and $\delta^{(n)}$ do not depend on probabilities, so the superscript $(n)$ can be dropped. In order to show that inequality (10) holds uniformly it suffices to choose $N_{1}$ large enough that $\sigma^{(n)}(1+1 / a)<1$ for all $n>N_{1}$. This is easily achievable since $p_{k}^{(n)} \rightarrow p_{k}$ for all $k=1, \ldots, K$. The uniform boundedness of 
$\left\{f^{(n)}\right\}$ in $B V$ and the invariance of its limit points under $P_{T}$ follow in a similar way to the one-dimensional case. Note that in this case it is not necessary to consider a higher power of the map $T^{(n)}$ as opposed to the one-dimensional case.

\section{Main Result}

Let $T=\left\{\tau_{1}, \tau_{2} ; p_{1}, p_{2}\right\}$ be an $N$-dimensional random map with position dependent probabilities $p_{1}(x)=1$ and $p_{2}(x)=0$ satisfying conditions of the previous section. Note that $T$ is essentially the same map as $\tau_{1}$. Suppose the domain of $T$ is $I=I_{1} \cup I_{2}$, where $I_{1}$ and $I_{2}$ are invariant under $\tau_{1}$. Suppose $\tau_{1}$ has exactly two ergodic measures $\mu_{1}$, and $\mu_{2}$ with densities $f_{1}$ and $f_{2}$ on $I_{1}$ and $I_{2}$, respectively. The map $\tau_{2}$ differs from $\tau_{1}$ on the sets $H_{1,2} \subset I_{1}$ and $H_{2,1} \subset I_{2}$, where $H_{1,2}=I_{1} \cap \tau_{2}^{-1}\left(I_{2}\right)$ and $H_{2,1}=I_{2} \cap \tau_{2}^{-1}\left(I_{1}\right)$. We assume that

$$
\mu_{1}\left(H_{1,2}\right)>0 \text { and } \mu_{2}\left(H_{2,1}\right)>0 \text {. }
$$

Now consider a sequence of random maps $T^{(n)}=\left\{\tau_{1}, \tau_{2} ; p_{1}^{(n)}, p_{2}^{(n)}\right\}$ as perturbations of $T$, where only the probabilities are changed. Let

$$
\begin{aligned}
& p_{2}^{(n)}=p_{2,1}^{(n)} \chi_{H_{2,1}}+p_{1,2}^{(n)} \chi_{H_{1,2}} \\
& p_{1}^{(n)}=1-p_{2}^{(n)},
\end{aligned}
$$

with $p_{1,2}^{(n)}, p_{2,1}^{(n)}>0$, independent of $x$. Our main result is the following theorem.

Theorem 6. If $p_{1,2}^{(n)}, p_{2,1}^{(n)} \rightarrow 0$ and $\lim _{n \rightarrow \infty} \frac{p_{2,1}^{(n)}}{p_{1,2}^{(n)}}$ exists, then the acim's of the $n$-dimensional random maps $T^{(n)}$ converge to the measure $\mu=\alpha_{1} \mu_{1}+\alpha_{2} \mu_{2}$, where

$$
\frac{\alpha_{1}}{\alpha_{2}}=\frac{\mu_{2}\left(H_{2,1}\right)}{\mu_{1}\left(H_{1,2}\right)} \lim _{n \rightarrow \infty} \frac{p_{2,1}^{(n)}}{p_{1,2}^{(n)}} .
$$

Proof. Let $\mu_{T^{(n)}}$ be an acim of $T^{(n)}$ (we do not assume its uniqueness). Let $f^{(n)}$ be the invariant density of $\mu_{T^{(n)}}$.

By Theorem 5, $\left\{f^{(n)}\right\}_{n \geq 1}$ is precompact in $L^{1}$ and if $f^{*}$ is a limit point, then $f^{*}$ is of the form $\alpha_{1} f_{1}+\alpha_{2} f_{2}$ for some $0 \leq \alpha_{1}, \alpha_{2} \leq 1, \alpha_{1}+\alpha_{2}=1$. In terms of the corresponding measures, there exists a subsequence $n_{k}$ such that:

$$
\begin{aligned}
& \mu_{T^{\left(n_{k}\right)}}\left(H_{1,2}\right) \rightarrow \alpha_{1} \mu_{1}\left(H_{1,2}\right)+\alpha_{2} \mu_{1}\left(H_{2,1}\right)=\alpha_{1} \mu_{1}\left(H_{1,2}\right) \\
& \mu_{T^{\left(n_{k}\right)}}\left(H_{2,1}\right) \rightarrow \alpha_{1} \mu_{2}\left(H_{1,2}\right)+\alpha_{2} \mu_{2}\left(H_{2,1}\right)=\alpha_{2} \mu_{2}\left(H_{2,1}\right)
\end{aligned}
$$

By (11), (14) and (15) we have $\mu_{T^{(n)}}\left(H_{1,2}\right)>0$ or $\mu_{T^{(n)}}\left(H_{2,1}\right)>0$. Let us assume $\mu_{T^{(n)}}\left(H_{2,1}\right)>0$ without loss of generality. Then,

$$
\begin{aligned}
\mu_{T^{(n)}}\left(I_{1}\right)=\int_{I} \mathbb{P}\left(x, I_{1}\right) d \mu_{T^{(n)}} & =1 \cdot \mu_{T^{(n)}}\left(I_{1} \backslash H_{1,2}\right)+\left(1-p_{1,2}^{(n)}\right) \cdot \mu_{T^{(n)}}\left(H_{1,2}\right) \\
& +0 \cdot \mu_{T^{(n)}}\left(I_{2} \backslash H_{2,1}\right)+p_{2,1}^{(n)} \mu_{T^{(n)}}\left(H_{2,1}\right) .
\end{aligned}
$$

Hence,

$$
\frac{\mu_{T^{(n)}}\left(H_{1,2}\right)}{\mu_{T^{(n)}}\left(H_{2,1}\right)}=\frac{p_{2,1}^{(n)}}{p_{1,2}^{(n)}} .
$$

Applying (16), (14) and (15), we get

$$
\frac{\alpha_{1}}{\alpha_{2}}=\frac{\mu_{2}\left(H_{2,1}\right)}{\mu_{1}\left(H_{1,2}\right)} \lim _{k \rightarrow \infty} \frac{p_{2,1}^{\left(n_{k}\right)}}{p_{1,2}^{\left(n_{k}\right)}} .
$$


Remark 4.1. If the probabilities $p_{1,2}^{(n)}, p_{2,1}^{(n)}$ depend on position, we obtain slightly more general formula

$$
\frac{\alpha_{1}}{\alpha_{2}}=\lim _{n \rightarrow \infty} \frac{\int_{H_{2,1}} p_{2,1}^{(n)} d \mu_{2}}{\int_{H_{1,2}} p_{1,2}^{(n)} d \mu_{1}},
$$

provided that the limit on the right hand side exists.

Additional information about the spectrum of operators $P_{T^{(n)}}$ is provided in the following theorem based on results of [Keller and Liverani, 1999].

Theorem 7. Let us assume that 1 is an eigenvalue of $P_{T}$ of multiplicity 2. For arbitrarily small $\delta>0$, there exists an $n_{\delta}$ such that for $n \geq n_{\delta}$ the spectrum of $P_{T^{(n)}}$ intersected with $\{z:|z-1|<\delta\}$ consists of two eigenvalues of multiplicity 1: 1 and $r_{n},\left|r_{n}\right| \leq 1, r_{n} \neq 1$ and $r_{n} \rightarrow 1$, as $n \rightarrow \infty$.

Proof. The family $P_{T^{(n)}}, n \geq 1$, satisfies the assumptions of Corollary 1 of [Keller and Liverani, 1999] which implies the above statement.

\section{Main theorem with $L$ ergodic components}

Let $T=\left\{\tau_{1}, \tau_{2} ; p_{1}, p_{2}\right\}$ be an $N$-dimensional random map with position dependent probabilities $p_{1}(x)=1$ and $p_{2}(x)=0$. So $T$ is essentially the same map as $\tau_{1}$. Suppose $\tau_{1}$ has $L$ ergodic components $I_{1}, \ldots, I_{L}$, $\cup_{i=1}^{L} I_{i}=I$. Suppose there are $L-1$ pairwise disjoint "holes" $\left\{H_{i, j}\right\}_{1 \leq j \leq L, j \neq i}$ in each component $I_{i}$. Map $\tau_{2}$ is defined as a piecewise expanding map which has the following properties

$$
\tau_{2}\left(H_{i, j}\right) \subset I_{j}, \text { for } i, j \in\{1, \ldots, L\}
$$

and $\tau_{2}=\tau_{1}$ outside the holes.

Let $T^{(n)}=\left\{\tau_{1}, \tau_{2} ; p_{1}^{(n)}, p_{2}^{(n)}\right\}$ be a sequence of random maps such that

$$
1-p_{1}^{(n)}=p_{2}^{(n)}=\sum_{i=1}^{L} \sum_{j \neq i} p_{i, j}^{(n)} \chi_{H_{i, j}}
$$

$0<p_{i, j}^{(n)}<1$ and

$$
p_{i, j}^{(n)}=h(n) a_{i, j}+o(h(n)),
$$

for some function $h$ such that $\lim _{n \rightarrow \infty} h(n)=0$. Let $\mu_{T}^{(n)}$ denote the invariant measure of $T^{(n)}$. Then for every $1 \leq k \leq L$,

$$
\mu_{T^{(n)}}\left(I_{k}\right)=\int \mathbb{P}\left(x, I_{k}\right) d \mu_{T^{(n)}}(x)=\int_{\tau_{1}^{-1}\left(I_{k}\right)} p_{1}^{(n)}(x) d \mu_{T^{(n)}}+\int_{\tau_{2}^{-1}\left(I_{k}\right)} p_{2}^{(n)}(x) d \mu_{T^{(n)}} .
$$

It follows that for every $1 \leq k \leq L$,

$$
\sum_{j \neq k} p_{k, j}^{(n)} \mu_{T^{(n)}}\left(H_{k, j}\right)=\sum_{i \neq k} p_{i, k}^{(n)} \mu_{T^{(n)}}\left(H_{i, k}\right) .
$$

The left hand side of the equation (19) can be interpreted as the amount of $\mu_{T^{(n)} \text {-measure that leaves }}$

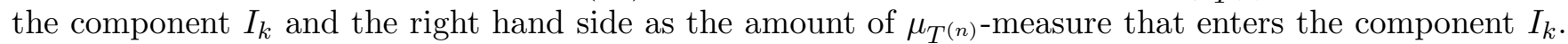
Intuitively, these two quantities are equal because $\mu_{T^{(n)}}$ is preserved under $T^{(n)}$.

Let us define $q_{i, j}=a_{i, j} \mu_{i}(H(i, j))$ for $j \neq i, q_{i, i}=1-\sum_{j \neq i} q_{i, j}$ for $1 \leq i \leq L$, and

$$
Q=\left[q_{i, j}\right]_{1 \leq i, j \leq L}
$$

By the continuity theorem for random maps, there exists a subsequence $n_{k}$ such that $\mu_{T^{\left(n_{k}\right)}} \rightarrow \mu_{T}=$ $\sum_{i=1}^{L} \alpha_{i} \mu_{i}$. Therefore, $\mu_{T^{\left(n_{k}\right)}}\left(H_{i, j}\right) \rightarrow \alpha_{i} \mu_{i}\left(H_{i, j}\right)$. Hence, the equations (19), for $n=n_{k}$, can be written as

$$
\sum_{j \neq k} a_{k, j} \alpha_{k} \mu_{k}\left(H_{k, j}\right)=\sum_{i \neq k} a_{i, k} \alpha_{i} \mu_{i}\left(H_{i, k}\right)+o(1)
$$


or

$$
\left(1-q_{k, j}\right) \alpha_{k}=\sum_{i \neq k} q_{i, k} \alpha_{i}+o(1)
$$

which in matrix form is

$$
\alpha Q=\alpha+o(1)
$$

where $\alpha=\left(\alpha_{1}, \ldots, \alpha_{L}\right)$. If, for $w=\left(w_{1}, \ldots, w_{L}\right)$ the solution of the equation $w=w Q$ is stable under small perturbations, then, $\alpha=\left(\alpha_{1}, \ldots, \alpha_{L}\right)$ satisfies

$$
\alpha \cdot Q=\alpha
$$

The conditions for stability of eigenvectors for probability matrices are well known, see for example [Cho and Meyer, 2001].

We proved the following theorem

Theorem 8. Let $T^{(n)}$ be a sequence of random maps satisfying assumptions of Section 4 but such that map $\tau_{1}$ has $L \geq 2$ ergodic components. Let probabilities $p_{i, j}^{(n)}, 1 \leq i, j \leq L$ satisfy assumptions (18). If the matrix $Q$ defined in (20) has stable left 1-eigenvector, then the invariant measures $\mu_{T^{(n)}}$ converge as $n \rightarrow \infty$ to the measure $\sum_{i=1}^{L} \alpha_{i} \mu_{i}$, where $\alpha Q=\alpha$, and $\mu_{i}$ is the $\tau_{1}$-invariant measure on the $i$-th ergodic component.

\section{Examples}

\section{Example 6.1.}

We now present a simple Markov map example on the interval [0,1]. Consider the maps $\tau_{1}$ and $\tau_{2}$ as shown in figure 1.

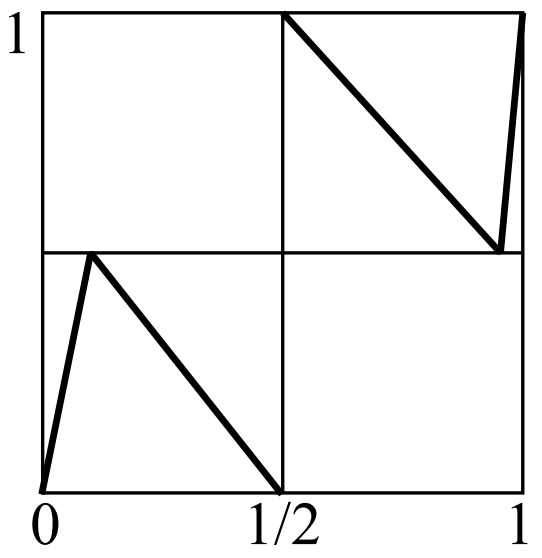

a)

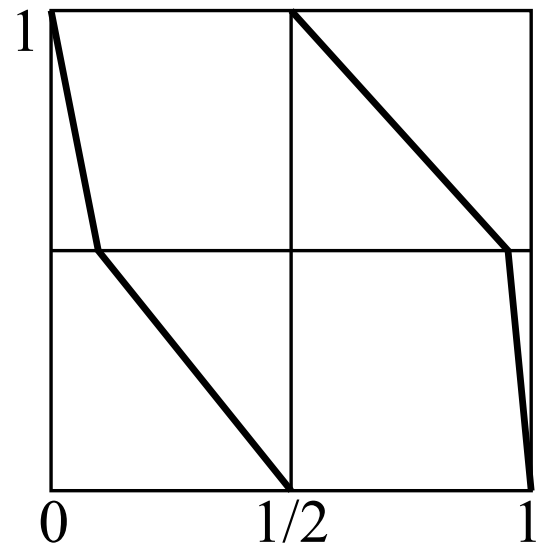

b)

Fig. 1. Maps $\tau_{1}$ and $\tau_{2}$.

Both maps are Markov on the partition $\mathcal{P}=\left\{J_{1}=[0,0.1], J_{2}=[0.1,0.5], J_{3}=[0.5,0.95], J_{4}=\right.$ $[0.95,1]\}$. Let $|J|$ denote the Lebesgue measure of the set $J$. Then $\left|J_{1}\right|=0.1,\left|J_{2}\right|=0.4,\left|J_{3}\right|=0.45$, $\left|J_{4}\right|=0.05 . \tau_{1}$ and $\tau_{2}$ have slopes of the same magnitude on $J_{1}, \ldots, J_{4}$. They are $s_{1}=5, s_{2}=5 / 4$, $s_{3}=10 / 9, s_{4}=10$, respectively. The ergodic components of $\tau_{1}$ are $I_{1}=J_{1} \cup J_{2}$ and $I_{2}=J_{3} \cup J_{4}$. The holes are $H_{1,2}=J_{1}$ and $H_{2,1}=J_{4}$.

Our aim is to compute the acims of the random maps $T=\left\{\tau_{1}, \tau_{2}, 1,0\right\}$ and $T^{(n)}=\left\{\tau_{1}, \tau_{2}, p_{1}^{(n)}, p_{2}^{(n)}\right\}$, where $p_{1}^{(n)}$ and $p_{2}^{(n)}$ are defined as in equation (13) and (12). To this end, we will first compute the invariant densities of $\tau_{1}$ and $\tau_{2}$. 
The matrices corresponding to Perron-Frobenius operators for $\tau_{1}$ and $\tau_{2}$ are

$$
M_{\tau_{1}}=\left[\begin{array}{cccc}
1 / 5 & 1 / 5 & 0 & 0 \\
4 / 5 & 4 / 5 & 0 & 0 \\
0 & 0 & 9 / 10 & 9 / 10 \\
0 & 0 & 1 / 10 & 1 / 10
\end{array}\right] \quad, \quad M_{\tau_{2}}=\left[\begin{array}{cccc}
0 & 0 & 1 / 5 & 1 / 5 \\
4 / 5 & 4 / 5 & 0 & 0 \\
0 & 0 & 9 / 10 & 9 / 10 \\
1 / 10 & 1 / 10 & 0 & 0
\end{array}\right] .
$$

Any invariant density of $\tau_{1}$ or $\tau_{2}$ is piecewise constant on the partition $\mathcal{P}$. Moreover, if we denote the value of the invariant density on $J_{i}$ by $f_{i}, 1 \leq i \leq 4$, then $\left(f_{1}, f_{2}, f_{3}, f_{4}\right)$ is the left eigenvector of the Perron-Frobenius matrix corresponding to eigenvalue 1 . For $\tau_{2}$, one easily checks that $(2 / 3,2 / 3,4 / 3,4 / 3)$ is the unique normalized invariant density. On the other hand, $\tau_{1}$ has two ergodic components with acims $\mu_{1}$ and $\mu_{2}$ which are simply the normalized Lebesgue measure on $I_{1}$ and $I_{2}$, respectively. Any acim of $\tau_{1}$ is of the form $t \mu_{1}+(1-t) \mu_{2}, 0 \leq t \leq 1$.

It follows from equation (6) that the invariant density of $T$ is the same as the invariant density of $\tau_{1}$.

For the random map $T^{(n)}=\left\{\tau_{1}, \tau_{2} ; p_{1}^{(n)}, p_{2}^{(n)}\right\}$, equation (6) implies that the invariant density $\left(f_{1}^{(n)}, f_{2}^{(n)}, f_{3}^{(n)}, f_{4}^{(n)}\right)$ satisfies

$$
\begin{aligned}
\left(f_{1}^{(n)}, f_{2}^{(n)}, f_{3}^{(n)}, f_{4}^{(n)}\right)= & \left(\left(1-p_{1,2}^{(n)}\right) f_{1}, f_{2}^{(n)}, f_{3}^{(n)},\left(1-p_{2,1}^{(n)}\right) f_{4}^{(n)}\right) M_{\tau_{1}} \\
& +\left(p_{1,2}^{(n)} f_{1}^{(n)}, 0,0, p_{2,1}^{(n)} f_{4}^{(n)}\right) M_{\tau_{2}}
\end{aligned}
$$

which yields $f_{1}^{(n)}=f_{2}^{(n)}, f_{3}^{(n)}=f_{4}^{(n)}$ and $p_{2,1}^{(n)} f_{4}^{(n)}=2 p_{1,2}^{(n)} f_{1}^{(n)}$. So the unique normalized invariant density for $T^{(n)}$ is

$$
f^{(n)}=\frac{2}{p_{2,1}^{(n)}+2 p_{1,2}^{(n)}}\left(p_{2,1}^{(n)}, p_{2,1}^{(n)}, 2 p_{1,2}^{(n)}, 2 p_{1,2}^{(n)}\right) .
$$

Suppose $\lim _{n \rightarrow \infty} p_{2,1}^{(n)} / p_{1,2}^{(n)}=l$. Then $f^{(n)} \rightarrow(2 /(2+l))(l, l, 2,2)$. It follows that the invariant measure $\mu_{T^{(n)}} \rightarrow \alpha_{1} \mu_{1}+\alpha_{2} \mu_{2}$, where $\alpha_{1}=(2 l) /(l+2)$ and $\alpha_{2}=4 /(l+2)$. Moreover,

$$
\frac{\alpha_{1}}{\alpha_{2}}=\frac{1}{2} l=\frac{0.05}{0.1} l=\frac{\mu_{2}\left(H_{2,1}\right)}{\mu_{1}\left(H_{1,2}\right)} \lim _{n \rightarrow \infty} \frac{p_{2,1}^{(n)}}{p_{1,2}^{(n)}} .
$$
$(21))$

The Perron-Frobenius operator for the random map $T^{(n)}$ corresponds to the matrix (already shown in

$$
M_{T^{(n)}}=\left[\begin{array}{cccc}
1 / 5-(1 / 5) p_{1,2}^{(n)} & 4 / 5 & 0 & (1 / 10) p_{1,2}^{(n)} \\
1 / 5 & 4 / 5 & 0 & 0 \\
0 & 0 & 9 / 10 & 1 / 10 \\
(1 / 5) p_{2,1}^{(n)} & 0 & 9 / 10 & 1 / 10-(1 / 10) p_{2,1}^{(n)}
\end{array}\right]
$$

with eigenvalues: $1, r_{1}^{(n)}=1 / 2-(1 / 20) p_{2,1}^{(n)}-(1 / 10) p_{1,2}^{(n)}+a, r_{2}^{(n)}=1 / 2-(1 / 20) p_{2,1}^{(n)}-(1 / 10) p_{1,2}^{(n)}-a^{(n)}$ and 0 , where

$$
a^{(n)}=(1 / 20) \sqrt{100+16 p_{2,1}^{(n)}+24 p_{1,2}^{(n)}+\left(p_{2,1}^{(n)}\right)^{2}+4 p_{2,1}^{(n)} p_{1,2}^{(n)}+4\left(p_{1,2}^{(n)}\right)^{2}}
$$

For $p_{1,2}^{(n)}$ and $p_{2,1}^{(n)}$ close to 0 , we have $r_{1}^{(n)}$ close to 1 and $r_{2}^{(n)}$ close to 0 . For example, if $p_{1,2}^{(n)}=$ $p_{2,1}^{(n)}=0.01$, then $r_{1}^{(n)} \sim 0.9995$ and $r_{2}^{(n)} \sim-0.0025$. The eigenvector corresponding to $r_{1}^{(n)}$ is $v \sim$ $[-0.749265,-0.751139,0.375571,0.373698]$.

\section{Example 6.2.}


We present a random map with 3 ergodic components of the original map $\tau_{1}$, see figure 2 . Consider maps $\tau_{1}$ and $\tau_{2}$ on a set $I=[0,1]: \tau_{1}$ has three ergodic components $I_{1}=[0,1 / 3], I_{2}=[1 / 3,2 / 3]$ and $I_{3}=[2 / 3,1]$, $\cup_{i=1,2,3} I_{i}=I$. On each components normalized Lebesgue measure $\mu_{i}, i=1,2,3$, is $\tau_{1}$-invariant. There are 2 holes in each component. They are

$$
\begin{aligned}
& H_{1,2}=[1 / 9,2 / 9], H_{1,3}=[2 / 9,1 / 3] \subset I_{1} ; \\
& H_{2,1}=[1 / 3,4 / 9], H_{2,3}=[5 / 9,2 / 3] \subset I_{2} ; \\
& H_{3,1}=[2 / 3,7 / 9], H_{3,2}=[7 / 9,8 / 9] \subset I_{3} .
\end{aligned}
$$
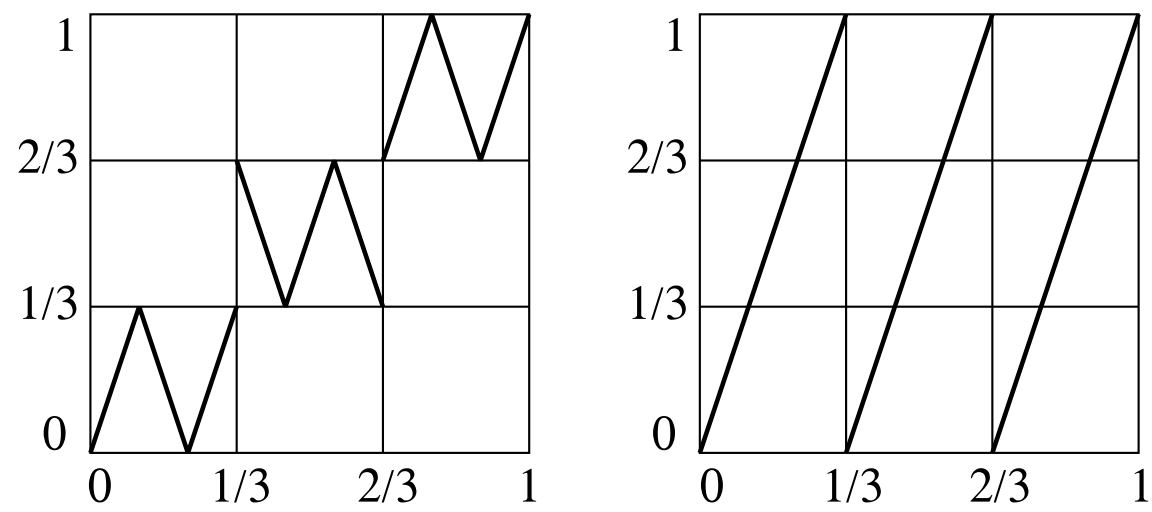

Fig. 2. Maps $\tau_{1}$ and $\tau_{2}$ for Example 6.2 with 3 ergodic components.

Map $\tau_{2}$ is defined as a piecewise expanding map shown in Fig. 2. It has the following properties

$$
\tau_{2}\left(H_{i, j}\right) \subset I_{j}, \text { for } i, j \in\{1,2,3\}
$$

and $\tau_{2}=\tau_{1}$ outside the holes.

We define the probabilities that each of the holes will be used by

$$
p_{i, j}^{(n)}=h(n) a_{i, j}+o(h(n)), \quad 1 \leq i, j \leq 3,
$$

where $h$ is such that $\lim _{n \rightarrow \infty} h(n)=0$ and the matrix $A=\left[a_{i, j}\right]_{1 \leq i, j \leq 3}$ is given by

$$
A=\left[\begin{array}{ccc}
0 & 0.3 & 0.5 \\
0.7 & 0 & 0.2 \\
0.1 & 0.1 & 0
\end{array}\right]
$$

The position dependent probability of applying the map $\tau_{2}$ is defined by

$$
p_{2}^{(n)}(x)=\sum_{i=1,2,3} \sum_{j \neq i} p_{i, j}^{(n)} \chi_{H_{i, j}}(x), \quad x \in I .
$$

The probability of applying map $\tau_{1}$ is defined by $p_{1}^{(n)}(x)=1-p_{2}^{(n)}(x), x \in I$.

Consider the random map $T^{(n)}=\left\{\tau_{1}, \tau_{2} ; p_{1}^{(n)}, p_{2}^{(n)}\right\}$. Let $\mu_{T^{(n)}}$ be its invariant measure. By the continuity theorem, $\mu_{T^{(n)}} \rightarrow \alpha_{1} \mu_{1}+\alpha_{2} \mu_{2}+\alpha_{3} \mu_{3}$ as $p_{i, j} \rightarrow 0, i \neq j$. Since $\mu_{i}\left(H_{i, j}\right)=1 / 3$ for $i \neq j, 1 \leq i, j \leq 3$, by (20) we have

$$
Q=\frac{1}{30}\left[\begin{array}{ccc}
22 & 3 & 5 \\
7 & 21 & 2 \\
1 & 1 & 28
\end{array}\right]
$$

Therefore, the normalized vector $\alpha=\left(\alpha_{1}, \alpha_{2}, \alpha_{3}\right)=\frac{1}{78}(16,11,51)$.

\section{Example 6.3.}


We consider a two dimensional Markov map example with $\tau_{1}$ having 4 ergodic components. A Maple 12 program with the details of the example is available on request. We will use the notation of Example 6.2. The space $I$ is a unit square of the plane $\mathbb{R}^{2}$. It is divided into 4 identical subsquares $I_{1}, I_{2}, I_{3}, I_{4}$ and each of them is further divided into 9 identical smaller subsquares: $I_{1}=\cup_{i=1}^{9} S_{i}, I_{2}=\cup_{i=10}^{18} S_{i}, I_{3}=\cup_{i=19}^{27} S_{i}$, $I_{4}=\cup_{i=28}^{36} S_{i}$, as in figure 6 .

\begin{tabular}{l|c|c|c|c|c|c|}
\hline 1 & 2 & 3 & 10 & 11 & 12 \\
\hline 4 & 5 & 6 & 13 & 14 & 15 \\
\hline 7 & 8 & 9 & 16 & 17 & 18 \\
\hline 19 & 20 & 21 & 28 & 29 & 30 \\
\hline 22 & 23 & 24 & 31 & 32 & 33 \\
\hline 25 & 26 & 27 & 34 & 35 & 36 \\
\hline
\end{tabular}$I_{2}$

Fig. 3. The Markov partition for map $\tau_{1}$ of Example 6.3.

We define $\tau_{1}$ restricted to each of $I_{i}, i=1,2,3,4$, as the same Markov map transforming each square $S_{j}$ onto four squares $S_{k}$ in such a way that the corresponding adjacency matrix of the map $\tau_{1}$ restricted to $I_{i}$ is

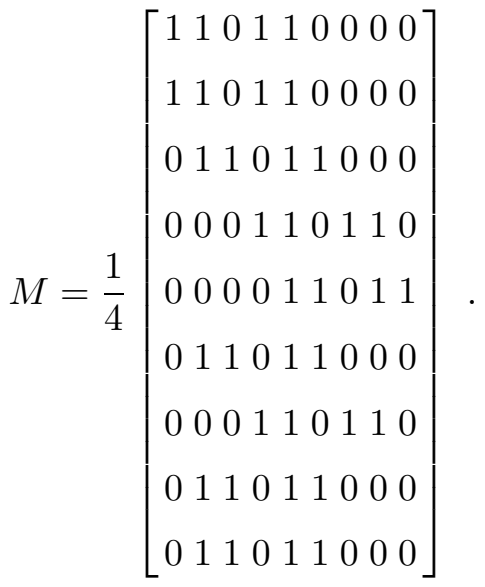

The matrix $M_{\tau_{1}}$ corresponding to $\tau_{1}$ is the block matrix with 4 matrices $M$ along the diagonal. The map $\tau_{1}$ has 4 ergodic components. For each component the normalized acim $\mu_{i}, i=1,2,3,4$, invariant for $\tau_{1}$ restricted to $I_{i}$, can be represented by the vector

$$
\begin{aligned}
& {\left[\mu_{i}(1), \mu_{i}(2), \mu_{i}(3), \mu_{i}(4), \mu_{i}(5), \mu_{i}(6), \mu_{i}(7), \mu_{i}(8), \mu_{i}(9)\right]} \\
& =[0.05357,0.16071,0.10714,0.08036,0.25,0.16964,0.02679,0.08929,0.0625] .
\end{aligned}
$$

The squares $S_{6}, S_{8}, S_{13}, S_{17}, S_{20}, S_{24}, S_{29}, S_{31}$, are designated as holes. We have $S_{6}=H_{1,2}, S_{8}=H_{1,3}$, $S_{13}=H_{2,1}, S_{17}=H_{2,4}, S_{20}=H_{3,1}, S_{24}=H_{3,4}, S_{29}=H_{4,2}, S_{31}=H_{4,3}$. We have $\mu_{1}\left(S_{6}\right)=\mu_{3}\left(S_{24}\right)=$ 0.16964, $\mu_{1}\left(S_{8}\right)=\mu_{2}\left(S_{17}\right)=0.08929, \mu_{2}\left(S_{13}\right)=\mu_{4}\left(S_{31}\right)=0.08036, \mu_{3}\left(S_{20}\right)=\mu_{4}\left(S_{29}\right)=0.16071$.

We define $\tau_{2}$ to be the Markov map on $I$ which realizes the transfers. On squares which are not holes it is equal to $\tau_{1}$. On each of the squares which is a hole $\tau_{2}$ is a linear map transferring this square onto four squares in appropriate component $I_{j}$. The matrix $M_{\tau_{1}}$ has most of its rows the same as the matrix $M_{\tau_{1}}$, except for rows 6,8,13,17,20,24,29,31 which have elements $(6,10),(6,11),(6,13),(6,14),(8,19),(8,20),(8,22),(8,23),(13,5),(13,6),(13,8),(13,9)$, 
$(17,29),(17,30),(17,32),(17,33),(20,4),(20,5),(20,7),(20,8),(24,31),(24,32),(24,34),(24,35),(29,14)$, $(29,15),(29,17),(29,18),(31,20),(31,21),(31,23),(31,24)$, equal to $1 / 4$ and all other elements 0.

Let $h$ be such that $\lim _{n \rightarrow \infty} h(n)=0$. We define the matrix of transfer probabilities between $I_{i}$ and $I_{j}$ as

$$
P^{(n)}=\left[p_{i, j}^{(n)}\right]_{1 \leq i, j \leq 4}=h(n) \cdot A \quad, \quad \text { where } \quad A=\left[\begin{array}{cccc}
0 & 0.4 & 0.5 & 0 \\
0.3 & 0 & 0 & 0.8 \\
0.7 & 0 & 0 & 0.5 \\
0 & 0.6 & 0.6 & 0
\end{array}\right] .
$$

We define position dependent probabilities $p_{1}^{(n)}, p_{2}^{(n)}$ as in (17). The random map $T^{(n)}=\left\{\tau_{1}, \tau_{2} ; p_{1}^{(n)}, p_{2}^{(n)}\right\}$ has matrix $M_{T^{(n)}}$ with rows the same as the rows of $M_{\tau_{1}}$ except for rows $6,8,13,17,20,24,29,31$ defined by

$$
\begin{aligned}
& \operatorname{row}\left(6, M_{T^{(n)}}\right)=\left(1-p_{1,2}^{(n)}\right) \operatorname{row}\left(6, M_{\tau_{1}}\right)+p_{1,2}^{(n)} \cdot \operatorname{row}\left(6, M_{\tau_{2}}\right), \\
& \operatorname{row}\left(8, M_{T^{(n)}}\right)=\left(1-p_{1,3}^{(n)}\right) \operatorname{row}\left(8, M_{\tau_{1}}\right)+p_{1,3}^{(n)} \cdot \operatorname{row}\left(8, M_{\tau_{2}}\right), \\
& \operatorname{row}\left(13, M_{T^{(n)}}\right)=\left(1-p_{2,1}^{(n)}\right) \operatorname{row}\left(13, M_{\tau_{1}}\right)+p_{2,1}^{(n)} \cdot \operatorname{row}\left(13, M_{\tau_{2}}\right), \\
& \operatorname{row}\left(17, M_{T^{(n)}}\right)=\left(1-p_{2,4}^{(n)}\right) \operatorname{row}\left(17, M_{\tau_{1}}\right)+p_{2,4}^{(n)} \cdot \operatorname{row}\left(17, M_{\tau_{2}}\right), \\
& \operatorname{row}\left(20, M_{T^{(n)}}\right)=\left(1-p_{3,1}^{(n)}\right) \operatorname{row}\left(20, M_{\tau_{1}}\right)+p_{3,1}^{(n)} \cdot \operatorname{row}\left(20, M_{\tau_{2}}\right), \\
& \operatorname{row}\left(24, M_{T^{(n)}}\right)=\left(1-p_{3,4}^{(n)}\right) \operatorname{row}\left(24, M_{\tau_{1}}\right)+p_{3,4}^{(n)} \cdot \operatorname{row}\left(24, M_{\tau_{2}}\right), \\
& \operatorname{row}\left(29, M_{T^{(n)}}\right)=\left(1-p_{4,2}^{(n)}\right) \operatorname{row}\left(29, M_{\tau_{1}}\right)+p_{4,2}^{(n)} \cdot \operatorname{row}\left(29, M_{\tau_{2}}\right), \\
& \operatorname{row}\left(31, M_{T^{(n)}}\right)=\left(1-p_{4,3}^{(n)}\right) \operatorname{row}\left(31, M_{\tau_{1}}\right)+p_{4,3}^{(n)} \cdot \operatorname{row}\left(31, M_{\tau_{2}}\right) .
\end{aligned}
$$

The $T^{(n)}$-invariant measure $\mu_{T^{(n)}}$ has been obtained using Maple. We define the vector $\alpha^{(n)}=$ $\left[\mu_{T^{(n)}}\left(I_{1}\right), \mu_{T^{(n)}}\left(I_{2}\right), \mu_{T^{(n)}}\left(I_{3}\right), \mu_{T^{(n)}}\left(I_{3}\right)\right]$. Then,

$$
\alpha^{(n)}=\frac{1}{126509}[25416,52668,14130,34295]+O(h(n)) .
$$

The matrix $Q$ is defined as in (20). The left 1-eigenvector of $Q$ is equal to $\lim _{n \rightarrow \infty} \alpha^{(n)}$.

For $\epsilon:=h(n)$ close to 0 the matrix corresponding to Frobenius-Perron operator of $T^{(n)}$ has, except 1, three other eigenvalues close to 1 but different from 1. For $\epsilon=10^{-3}$ they are $0.9997176900,0.9998399077$ and 0.9998924535. For $\epsilon=10^{-4}$ we obtained 0.9999717673, 0.9999839914, 0.9999892419.

Acknowledgment: The authors are grateful to anonymous reviewers for a detailed and constructive critique of the first version of the paper. The authors thank Gary Froyland for suggesting Remark 4.1.

\section{References}

Bahsoun, W. and Góra, P. [2005], Position Dependent Random Maps in One and Higher Dimensions, Studia Math. 166 (2005), 271-286.

Boyarsky, A. and Góra, P. [1997], Laws of Chaos, Birkhäuser, Boston, 1997.

Cho G.E. and Meyer C.D. [2001], Comparison of perturbation bounds for the stationary distribution of a Markov chain, Linear Algebra and its Applications 335 (2001), 137-150.

Dolgopyat, D. and Wright, P. [2011], The Diffusion Coefficient For Piecewise Expanding Maps Of The Interval With Metastable States, preprint arXiv:1011.5330.

Giusti, E. [1984], Minimal Surfaces and Functions of Bounded Variations, Birkhäuser, Boston, 1984.

Góra, P. and Boyarsky, A. [2003], Absolutely continuous invariant measures for random maps with position dependent probabilities, Math. Anal. Appl. 278 (2003), 225-242. 
Góra, P. and Boyarsky, A. [1989], Absolutely continuous invariant measures for piecewise expanding $C^{2}$ transformations in $\mathbb{R}^{N}$, Israel Jour. Math. 67, No. 3, (1989), 272-286.

Keller, G. and Liverani, C. [1999], Stability of the spectrum for transfer operators, Ann. Scuola Norm. Sup. Pisa Cl. Sci. (4), 28 (1)(1999), 141-152.

Gonzáles-Tokman, C., Hunt, B. R. and Wright, P. [2011], Approximating invariant densities of metastable systems, arXiv:0905.0223v1 [math.DS], Ergodic Theory and Dynamical Systems 31 (2011), pp. 13451361, doi:10.1017/S0143385710000337. 